\begin{tabular}{|c|c|c|c|c|c|c|}
\hline & $\begin{array}{l}\text { Group A } \\
\text { (INR } 11.4) n=25\end{array}$ & $\begin{array}{l}\text { Group B } \\
(\text { INR } 1.5-1.8) n=32\end{array}$ & $\begin{array}{l}\text { Group C } \\
\text { (INR } 1.9 \text { and above) } n=1\end{array}$ & $\begin{array}{l}\mathrm{p} \text { value } \\
\text { (group A vs B) }\end{array}$ & $\begin{array}{l}\text { p value } \\
\text { (group A vs C) }\end{array}$ & $\begin{array}{l}\text { p value } \\
\text { (group B vs C) }\end{array}$ \\
\hline Blood staining (\%) & 0 & 3.1 & 10 & 1.00 & 0.30 & 0.45 \\
\hline Hypotension (\%) & 20 & 9.4 & 30 & 0.28 & 0.66 & 0.13 \\
\hline Perforation (\%) & 0 & 0 & 0 & 1.00 & 1.00 & 1.00 \\
\hline Leaking site (\%) & 0 & 3.1 & 0 & 1.00 & 1.00 & 1.00 \\
\hline Death (\%) & 0 & 0 & 0 & 1.00 & 1.00 & 1.00 \\
\hline Peritonitis (\%) & 4 & 0 & 0 & 0.44 & 1.00 & 1.00 \\
\hline Infection (\%) & 0 & 3.1 & 0 & 1.00 & 1.00 & 1.00 \\
\hline
\end{tabular}

Conclusion The complication rate does not significantly increase with increasingly deranged coagulation. This is despite very low usage of FFP in this study. There is a trend towards an increased risk of bloodstaining and hypotension in the high INR group, which does not achieve statistical significance.

Disclosure of Interest None Declared.

\section{PWE-156 DURHAM METRICS TO EVALUATE EFFECTIVENESS OF A NEW CARE PATHWAY FOR PATIENTS WITH ADVANCE STAGE LIVER DISEASE (ASLD)}

'S Saksena*, ${ }^{2}$ L Hammal, ${ }^{2} \mathrm{C}$ Lancaster, ${ }^{2} \mathrm{M}$ Hewett. ${ }^{1}$ Hepatology, Durham, UK; ${ }^{2}$ County Durham Darlington Foundation Trust, Durham, UK

\subsection{6/gutjnl-2014-307263.416}

Introduction Patients with chronic liver disease have several unplanned admissions during their disease trajectory. ${ }^{1}$ We undertook a service improvement initiative to develop a new care pathway for patients with ASLD.

Methods Six month pilot of 20 ASLD patients, $(\geq 2$ admissions in last 12 months/'would you be surprised question' with 6-12 mths prognosis/Childs C). Team of hepatologist, community matron, hepatology nurse specialist and service improvement facilitator developed 'Durham Metrics': quantitative and qualitative metrics (Figure) to evaluate ASLD pathway, on best practice, ${ }^{2}$ further refined with focussed discussion with stakehoders. Results The metrics demonstrated that patient expereince prepilot was poor with multiple unplanned admissions and/or long

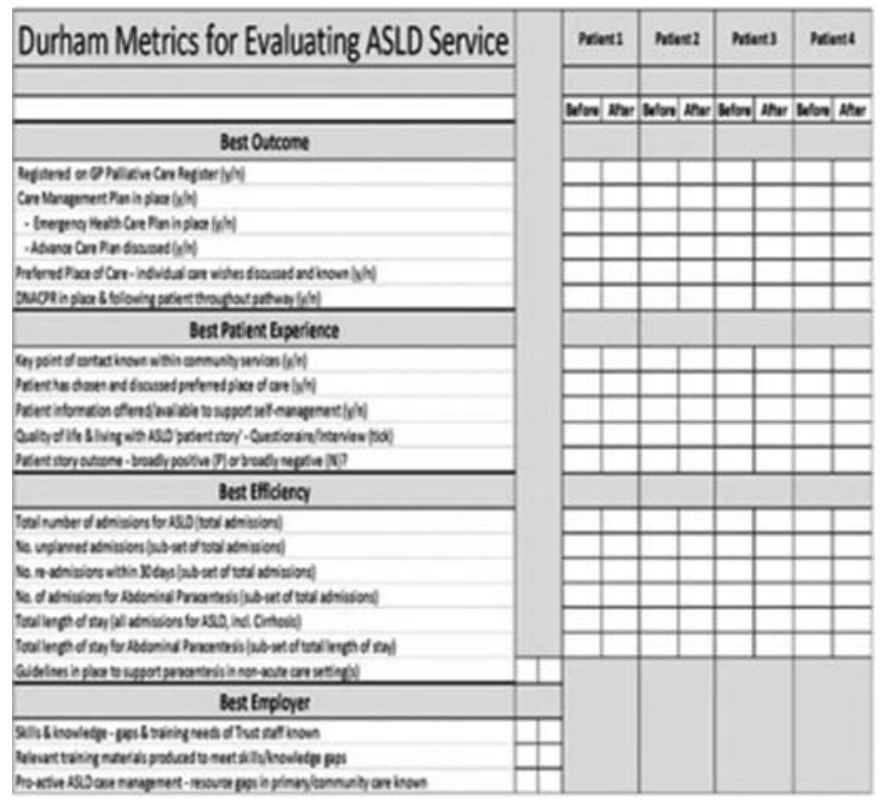

\section{Abstract PWE-156 Figure 1}

waits, preferred place of death was not discussed; care was not co-ordinated, and quality of life was often poor as a result. All post-pilot metrics reported significant improvements. Use of alternative community services, and shared care plans led to improved efficiency. $83 \%$ achieved their preferred place of care and death contrast to nil pre pilot.

Conclusion Key metrics of performance are essential to evaluate service improvement project. The project metrics designed for this project were able to capture changes initiated by pathway however more data and time is needed to draw statistically valid conclusions.

\section{REFERENCES}

1 National End of Life Care Intelligence Network 2012

2 Deciding Right

3 Liver Quest

Disclosure of Interest None Declared.

\section{PWE-157 EARLY TIPS (TRANSJUGULAR INTRAHEPATIC PORTOSYSTEMIC SHUNT) FOR ACUTE VARICEAL BLEEDING COMPLICATING ALCOHOLIC HEPATITIS (AH)}

'S Alam*, 'E Britton, ${ }^{2} U$ Shaikh, ${ }^{2} \mathrm{E}$ Evans, 'P Richardson. 'Gastroenterology, Royal Liverpool University Hospital, Liverpool, UK; ${ }^{2}$ Radiology, Royal Liverpool University Hospital, Liverpool, UK

\subsection{6/gutjnl-2014-307263.417}

Introduction The ideal management of variceal bleeding in the setting of acute alcoholic hepatitis is unclear. We present the outcome of this subgroup of patients in a cohort of patients treated with primary TIPS for variceal bleeding.

Methods A retrospective analysis on patients who had TIPS procedure performed as a primary treatment modality within $72 \mathrm{~h}$ of acute variceal bleeding from December 2010 to April 2013 with a minimum of 6 months follow up was performed.

Results 56 patients were included into the final analysis. In AH patients $(\mathrm{n}=18)$ mean age was 48 years $(30-65)$, mean discriminant function (DF) was 51 (24-87) and mean MELD score was 22. The 6 month mortality was $50 \%(9 / 18)$ with (7/9) dying within 30 days. The median HVPG $(\mathrm{mmHg})$ pre-TIPS and postTIPS were 16.5 and 6.5 respectively. In non-AH patient $(\mathrm{n}=$ 38) average age was 51y (25-70) mean MELD score was 14 (22-7). The mortality was $13 \%(5 / 38)$ at 6 months, (3/5) died by day 30 . The median HVPG $(\mathrm{mmHg})$ pre-TIPS and post-TIPS was 23 and 10 respectively.

Conclusion In patients with variceal bleeding complicating $\mathrm{AH}$ there is a higher 30 day and 6 month mortality in patients managed with a primary TIPS in comparison to patients with cirrhosis. The ideal management of this complex group remains unclear.

Disclosure of Interest None Declared. 